\title{
BURNOUT Y SATISFACCIÓN CON LA VIDA EN DOCENTES QUE REALIZAN CLASES VIRTUALES EN UN CONTEXTO DE PANDEMIA POR COVID-19
}

\author{
BURNOUT AND LIFE SATISFACTION IN TEACHERS TEACHING \\ VIRTUAL CLASSES IN A CONTEXT OF THE COVID-19 PANDEMIC \\ (Di) José Celio Pillaca ${ }^{*}$ \\ jose.celio@unmsm.edu.pe \\ ${ }^{1}$ Universidad Nacional Mayor de San Marcos, Lima, Perú \\ *Correspondencia: José Celio Pillaca. Email: jose.celio@unmsm.edu.pe
}

Recibido: 22.11.2020 | Aprobado: 16.12.2020

\section{RESUMEN}

El presente estudio tuvo por objetivo determinar la relación entre el Síndrome de Burnout y la Satisfacción con la Vida en docentes de Educación Básica Regular que impartían clases virtuales durante la pandemia por covid-19 en diversos departamentos del Perú. Bajo una metodología cuantitativa, de diseño descriptivo-correlacional, se aplicó de forma virtual el Inventario de Burnout de Maslach - Forma Ed (MBI-Es) y la Escala de Satisfacción con la vida (SWLS) a 56 docentes, quienes fueron seleccionados mediante un muestreo no probabilístico intencional. Se realizó el análisis descriptivo e inferencial de los datos mediante el programa estadístico SPSS versión 22 y se encontró que la mayor cantidad de docentes se sienten muy satisfechos con la vida, presentan niveles altos de Agotamiento Emocional, niveles bajos de Despersonalización y niveles altos de Realización Personal. Se hallaron diferencias significativas $(p<.05)$ según el sexo solo en la variable Despersonalización, y se encontró una relación estadísticamente significativa, baja y positiva $(\mathrm{p}<.05, \mathrm{r}=, 323)$ entre la Realización Personal y la Satisfacción con la Vida. Palabras clave: Covid-19, clases virtuales, síndrome de burnout, satisfacción con la vida.

\section{ABSTRACT}

The present study aimed to determine the relationship between the Burnout Syndrome and Satisfaction with Life in Regular Basic Education teachers who taught virtual classes during the COVID-19 pandemic in various departments of Peru. Under a quantitative methodology, with a descriptive-correlational design, the Maslach Burnout Inventory - Forma Ed (MBI-Es) and the Satisfaction with Life Scale (SWLS) were applied virtually to 56 teachers, who were selected by means of an intentional non-probability sampling. The descriptive and inferential analysis of the data was carried out using the SPSS version 22 statistical program and it was found that the largest number of teachers feel very satisfied with life, have high levels of Emotional Exhaustion, low levels of Depersonalization and high levels of Achievement. Personal. Significant differences (p $<.05$ ) were found according to sex only in the Depersonalization variable, and a statistically significant, low and positive relationship $(\mathrm{p}<.05, \mathrm{r}=.323)$ was found between Personal Achievement and Satisfaction with Lifetime.

Keywords: Covid-19, Virtual classes, Burnout Syndrome, Satisfaction with Life.

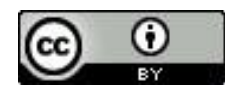




\section{INTRODUCCIÓN}

A finales del 2019 apareció en la ciudad de Wuhan (China) un brote a gran escala del síndrome respiratorio agudo-severo por coronavirus (SARS-Cov-2) o también llamado covid-19, el cual ha ido extendiéndose de forma progresiva a una gran mayoría de países en el mundo. Según el Centro europeo para la prevención y el control de enfermedades (2020) solo hasta el 20 de Noviembre de 2020 se han reportado más de 55 millones de casos positivos en el mundo y más de un millón de personas fallecidas a causa de este virus.

Según la Organización mundial de la salud (OMS, 2020), esta nueva enfermedad afecta a los tres componentes de salud (física, psicológica y social) y a todos los niveles sociodemográficos. En consecuencia, los gobiernos nacionales del mundo, como medida de afrontamiento ante esta nueva enfermedad, decretaron un proceso de confinamiento de sus ciudadanos, así como la suspensión o reducción de personal de algunos sectores laborales durante los primeros meses, a causa de los primeros casos positivos de covid-19 en sus países.

Es así que el estar confinado por varios meses en un mismo lugar, restringido a salir de casa más solo por emergencias, ha provocado que en las personas se desarrollen síntomas de malestar emocional y dificultades en su adaptación, tales como estrés, insatisfacción, problemas de sueño, ansiedad, miedo, preocupación, entre otros (González, Tejeda, Espinosa y Oliveros, 2020; Sandín, Valiente, García-Escalera y Chorot, 2020).

Sin embargo, paralelo a estas dificultades de adaptación psicológica y conductual, también está la proliferación del uso de las tecnologías en los ámbitos laborales, primordialmente en el sector educación. A causa de la suspensión de las clases presenciales en los colegios y universidades del mundo, las Tecnologías de la Información y la Comunicación (TIC) se han convertido en la herramienta indispensable para el docente, con el fin de continuar con las clases de una forma no presencial (virtual), y como una nueva oportunidad para innovar en la metodología de la enseñanza escolar (De Mendoza, 2020; Quispe, 2020).

Con respecto al Perú, se han tenido algunos antecedentes con miras a futuro sobre la incorporación de las TIC's a la educación y a la capacitación de los docentes en el uso de éstas (Hernández, 2017; Mateus y Suárez-Guerrero, 2017), no obstante, en la actualidad aún es incierto la cantidad de profesores que estén realmente capacitados con el uso de las TIC's. Una situación similar se ha encontrado en Paraguay durante la pandemia por covid-19 (véase Picón, Gonzáles y Paredes, 2020).

En este sentido, semanas después de decretarse la suspensión de las clases presenciales en todas las regiones del Perú, el Ministerio de Educación (MINEDU, 2020) implementó el programa Aprendo en Casa, dirigido a los estudiantes de colegios públicos del país a través de internet, radio y televisión, contando con una programación para Educación Básica Regular, Educación Especial y Educación Alternativa. Con respecto a los colegios particulares, se implementaron las clases no presenciales mediante el uso de plataformas virtuales como Zoom, Meet, Youtube, Whatsapp, EdModo, Cubicol, etc., al igual que otros países de América del Sur (Britez, 2020), esto demandaba una mayor 
inversión de tiempo para las capacitaciones en educación virtual, tanto a profesores, estudiantes y apoderados, lo cual afectaba a la vez el área familiar y laboral de los involucrados (Hidalgo, 2020; Mariátegui, 2020; Montes, 2020).

Al ser compleja esta adaptación a la nueva modalidad no presencial o de teletrabajo, los docentes están propensos a desarrollar un desgaste psíquico, manifestado a través de síntomas fisiológicos, poniendo en riesgo la enseñanza de sus alumnos. Además, se sabe que el estar expuesto a condiciones laborales deficientes, mantener malas relaciones con los padres y el tener estudiantes con problemas de conducta son principales factores de estrés en los profesores (Aloe, Shisler, Norris, Nickerson y Tyler, 2014; Skaalvik, E.M. y Skaalvik, S., 2007). Es por ello la importancia de este estudio, el cual permitirá conocer la situación actual de los profesores de Educación Básica Regular del Perú con respecto al Síndrome de Burnout y su posible relación con la satisfacción con la vida, con el fin de marcar un precedente para investigaciones futuras con respecto al tema en beneficio de la educación del país, mediante el cuidado del Bienestar Psicológico de sus maestros.

\section{Síndrome de Burnout}

El término Burnout fue acuñado a inicios de los 70 por el Dr. Freudenberg (1974, citado por Fernández, 2002), para detallar y explicar por qué los trabajadores del centro de salud en donde él trabajaba empezaban a sentirse cansados o trataban a sus pacientes con cierta indiferencia. A partir de entonces, el síndrome de Burnout ha sido explicado por varias teorías. Gil-Monte y Peiró (1999) nos mencionan tres modelos principales: 1) Modelos de la teoría sociocognitiva del yo, la cual postula que los pensamientos e ideas influyen en las emociones y conductas de las personas, pero que a su vez están influidas por estas acciones. El modelo indica también que el nivel de seguridad o confianza en las propias capacidades establece el esfuerzo que la persona dedique a la obtención de sus metas, así como la facilidad o dificultad para conseguirlas. 2) Modelos de la teoría del intercambio social, la cual propone que si el trabajador de una organización siente que su esfuerzo es desvalorado o que se le brinda un trato desigual ante los demás, posiblemente desarrollará el síndrome de Burnout; y 3) Modelos de la teoría organizacional, la cual da prioridad a los factores desencadenantes de estrés dentro y fuera del lugar de trabajo, como los problemas de rol (ej. el modelo de Golembiewski), la falta de temáticas de salud en la empresa (ej. el modelo de Cox, Kuk y Lwiter) y la poca importancia que se le da a la estructura, la cultura y el clima organizacional (ej, el modelo de Winnubst).

A nivel académico, la conceptualización más aceptada es según el modelo psicosocial de Maslach (2009), quien la define como un "síndrome psicológico que implica una respuesta prolongada a estresores interpersonales crónicos en el trabajo" (p. 37), manifestada a través de sus tres componentes: 1) Agotamiento Emocional, considerado el indicador fundamental del Burnout, en la cual la persona se sobreexige aún sin tener los recursos emocionales necesarios para afrontar la situación, sintiéndose fatigado y con bajas energías a causa de la sobrecarga laboral. 2) Despersonalización, regida por las relaciones interpersonales, en la cual la persona reacciona negativamente hacia quienes atiende, mostrándose insensible o indiferente con ellas, perjudicando su desempeño y su calidad de trabajo conforme pasa el tiempo; y 3) Realización Personal, en la cual la persona se siente autoeficaz, competente y con sentido de logro hacia el éxito profesional. Por lo cual se deja claro que la persona que ha desarrollado el Síndrome de Burnout evidencia 
niveles altos de Agotamiento Emocional y de Despersonalización, y niveles bajos de Realización Personal.

Al revisar la literatura se encuentran gran cantidad de estudios científicos que evalúan el Síndrome de Burnout en profesores de educación básica regular. En ese sentido, se sabe que en los docentes la Satisfacción Laboral se relaciona de forma inversa con el Agotamiento emocional (Arias, Sánchez y Ceballos, 2017; Cordero y Vargas, 2019; Mengui, 2018) y con la Despersonalización (Cordero y Vargas, 2019; Malander, 2016; Mengui, 2018), pero de forma positiva con la Realización Personal (Malander, 2016; Mengui, 2018). Así mismo, se tienen evidencias de que a mayor Agotamiento emocional presente el docente menor será su desempeño laboral (Achiri, 2019), también de que si la Despersonalización aumenta el desempeño docente disminuye (Achiri, 2019; Nayda, 2018), y que si aumenta la Realización Personal aumenta la autoeficacia (Mengui, 2018) y el desempeño docente (Achiri, 2019; Nayda, 2018).

A nivel nacional, el estudio del Burnout en los docentes ha sido muy prolífico, por ejemplo, en Arequipa los profesores que trabajaban en colegios nacionales presentaban mayor Despersonalización que los que trabajaban en colegios particulares, sin embargo, éstos últimos presentaban menor Realización Personal que aquellos docentes de colegios nacionales (Arias y Jiménez, 2013), también que los profesores varones presentaban mayor Agotamiento Emocional que las mujeres y que los docentes de Educación Primaria presentaban mayor Realización Personal que aquellos docentes que laboraban en Educación Inicial y Educación Secundaria (Arias, Huaman y Ceballos, 2019); en Iquitos se halló que la mayoría de los profesores de nivel primaria y secundaria presentaban niveles altos de Despersonalización, y niveles bajos en Agotamiento Emocional y Realización Personal (Pereira y Amacifen, 2018). En Lima, se halló que el Agotamiento Emocional, la Despersonalización y la Realización Personal se relacionan significativamente con la empatía (Romero, 2019). En Ayacucho, los maestros de esa región presentan altos niveles de Realización Personal y bajos niveles de Despersonalización y de Agotamiento Emocional, así mismo, se conoció que estos componentes no guardan relación con la Satisfacción con la Vida (Cárdenas, 2019).

\section{Satisfacción con la vida}

En la filosofía, hay dos perspectivas de Bienestar: el Hedónico, bajo el marco de Epicuro, y el Eudaimónico, basado en los postulados de Aristóteles. En Psicología, se estudia de manera científica las anteriores perspectivas con diferente terminología: Bienestar Subjetivo y Bienestar Psicológico. El primero se refiere a la evaluación afectiva y cognitiva de nuestra propia vida, conformado por los afectos positivos (emociones placenteras), los afectos negativos (emociones desagradables) y la satisfacción con la vida (Diener, Lucas y Oshi, 2004, citado por Morán, Vicente, Sánchez y Montes, 2014); y el segundo, se entiende como el estado de funcionamiento óptimo de la persona, la cual presenta varios modelos teóricos que van desde Jahoda (1958, en Lluch, 1999) con el modelo de salud mental positiva, hasta el reciente modelo de Seligman (2014), quien la define en base a cinco componentes: emociones positivas, compromiso, relaciones interpersonales positivas, el sentido de la vida y los logros alcanzados (modelo PERMA). 
Con lo que respecta a la satisfacción con la vida, ésta es entendida como el componente cognitivo del Bienestar Subjetivo, en donde el grado de satisfacción que tiene la persona con su propia vida se da mediante un juicio evaluativo de su calidad de vida y de los logros alcanzados según sus propios criterios personales (Pavot y Diener, 1993).

Gracias a los pocos estudios realizados sobre el tema, sabemos que, en Rumania por ejemplo, los profesores con bajos niveles de satisfacción con la vida eran propensos a sentirse emocionalmente agotados y a incumplir con sus deberes, también se halló que aquellos maestros que presentaban niveles bajos de inteligencia emocional eran más propensos a desarrollar el síndrome de Burnout, por último, se reportaron relaciones inversas entre el Síndrome de Burnout y las variables Inteligencia Emocional y Satisfacción con la Vida (Colomeischi, 2015). Un estudio más actual y cercano al Perú fue realizado por Sylva (2019) en Ecuador, quien encontró que la mayoría de las profesoras de educación Inicial se sentían muy satisfechas con la vida, así mismo, la autora halló que a medida que aumenta la edad de las docentes aumenta también la satisfacción con la vida.

A nivel Nacional se tienen estudios más actuales, en Ayacucho por ejemplo, Cárdenas (2019) encontró que la mayoría de los profesores de dicha provincia se sienten muy satisfechos con la vida. Resultados similares fueron reportados por Condori (2019), quien halló que la mayoría de los profesores de cuatro colegios de Tacna se sentían altamente satisfechos con la vida.

Sin embargo, ante todo lo dicho anteriormente, aún no se tienen antecedentes de investigaciones realizadas durante la pandemia por covid-19, con respecto al Burnout y la Satisfacción con la vida en los profesores de Educación Básica Regular, realizados de forma virtual. Es por las razones expuestas líneas arriba, que el presente estudio busca identificar si el síndrome de Burnout guarda alguna relación con la satisfacción con la vida en los docentes que realizan clases virtuales durante la pandemia por covid-19. Así mismo, el estudio presenta también los siguientes objetivos específicos: 1) describir los niveles del síndrome de Burnout y la Satisfacción con la Vida de los docentes y 2) conocer las diferencias según variables sociodemográficas en los temas de estudio. Considerando estos factores, la presente investigación es relevante y necesaria para la mejora de la educación en el país, por lo que los resultados de este estudio aportarían a las acciones necesarias para dicho fin.

\section{METODOLOGÍA}

Se empleó un tipo de investigación cuantitativa, de diseño descriptivo-correlacional, pues se buscó describir y relacionar las variables de estudio; de corte transversal, por ser la aplicación de los instrumentos en un momento único; y de análisis comparativo, por buscar diferencias según grupos de variables (Salgado, 2018).

\section{Participantes}

La población estuvo conformada por 493766 profesores de Educación Básica Regular de todo el Perú (Instituto Nacional de Estadística e Informática [INEI], 2017). La muestra fue seleccionada mediante un muestreo no probabilístico intencional (Salgado, 2018) y estuvo conformada por un total de 56 profesores que brindaban clases virtuales en colegios nacionales $(66,1 \%)$ y particulares $(33,9 \%)$, entre varones $(35,7 \%)$ y mujeres 
$(64,3 \%)$, del nivel Inicial $(10,7 \%)$, Primaria $(33,9 \%)$ y Secundaria $(55,4 \%)$, con edades comprendidas entre los 24 y los 63 años (media $=42,05$; DS=10,11), que trabajaban en Lima $(55,4 \%)$ y en provincias $(46,6 \%)$, ésta última conformada por docentes que laboraban en Ancash (14,3\%), Ica (5,4\%), Lambayeque (1,8\%), Madre de Dios (1,8\%), Junín (3,6\%), Tacna (1,8\%), Ayacucho (1,8\%), Huancavelica (3,6\%), Cajamarca $(1,8 \%)$, San Martín (1,8\%), Huánuco (3,6\%), Cusco (1,8\%) y Pasco (1,8\%).

Instrumentos de recolección de datos

\section{1) Inventario de Burnout de Maslach - Forma Ed (MBI-Es)}

Dirigido a profesores de Educación Básica y de Educación Superior. Compuesto por 22 ítems con siete opciones de respuesta, de aplicación individual y colectiva, con una duración de entre 10 a 15 minutos aproximadamente. El instrumento evalúa el síndrome de Burnout mediante sus tres componentes: Agotamiento Emocional, Despersonalización y Realización Personal. Creado por Cristina Maslach en Estados Unidos y adaptado al Perú por Fernández (2002), en docentes de primaria, quien halló una adecuada validez y alta confiabilidad para cada dimensión: Agotamiento Emocional $(\alpha=0,78)$, Despersonalización $(\alpha=0,76)$, y Realización Personal $(\alpha=0,74)$. En el presente estudio el instrumento contó con una buena validez de constructo, pues la medida de adecuación muestral de Kaiser-Meyer-Olkin (KMO) fue de ,747; el test de esfericidad de Bartlett fue significativo $\left(X^{2}=659,379 ; \mathrm{gl}=231\right.$ ) y mediante la técnica de componentes principales se extrajo 3 factores, que en conjunto explicaban el 52,501\% de la varianza total, cuyos ítems presentaban cargas factoriales mayores a .30. Con respecto a la confiabilidad del instrumento, se encontraron alta confiabilidades en las dimensiones Agotamiento Emocional $(\alpha=0,907)$ y Despersonalización $(\alpha=0,770)$, solo en la dimensión Realización el alfa de Cronbach fue de 0,688.

\section{2) Escala de Satisfacción con la Vida (SWLS)}

El instrumento, de escala likert, está compuesto por 5 ítems y conformado por 5 opciones de respuesta, de aplicación individual y colectiva, con una duración de 5 minutos aproximadamente. Evalúa el grado de satisfacción con la vida de la persona adulta. Cuenta con una puntuación mínima de 5 y una puntuación máxima de 25, a mayor puntuación mayor satisfacción con la vida. Creado por Diener, Emmons, Larsen y Griffin (1985) en Estados Unidos y adaptado al idioma español por Vázquez, Duque y Hervás (2013), quienes hallaron una adecuada validez y una alta confiabilidad $(\alpha=0,88)$. Fue validado al Perú por Calderón, Jozano, Cantuarias y Ibarra (2018), quien halló una alta consistencia interna $(\omega=0.90)$ en población adulta. En el presente estudio el instrumento contó con una buena validez de constructo, pues la medida de adecuación muestral de Kaiser-MeyerOlkin (KMO) fue de ,827; el test de esfericidad de Bartlett fue significativo $\left(\mathrm{X}^{2}=120,650\right.$; $\mathrm{gl}=10)$ y mediante la técnica de componentes principales se obtuvo un solo factor que explicaba el 64,198\% de la varianza total, cuyos ítems presentaban cargas factoriales mayores a .80 . Con respecto a la confiabilidad del instrumento, se obtuvo un alfa de Cronbach de 0,858 .

\section{Procedimiento}

El ítem 16 del MBI-Es (Trabajar en contacto directo con los alumnos, me produce bastante estrés) fue modificado por: "Trabajar en contacto directo con los alumnos, 
mediante una clase virtual, me produce bastante estrés", con el fin de mejorar la comprensión por parte de los docentes, siendo apropiado para el contexto de estudio. Luego, los instrumentos se convirtieron a un formato virtual utilizando el formulario de Google Drive. Se realizó una lista de grupos de Facebook de docentes de educación básica regular, en las cuales se publicó el enlace del formulario, la cual contenía el consentimiento informado y la explicación de la importancia del estudio. Se respetó la confidencialidad de los datos y la participación de los docentes fue de manera voluntaria. Los datos fueron recolectados durante los meses de Junio, Julio y Agosto del 2020. Una vez obtenida la información en el formulario Google Drive, se procedió a descargarla en un formato Excel. Dicha matriz de datos, obtenidos inicialmente en palabras, fueron convertidas a números, con el fin de ser utilizados en el programa estadístico SPSS versión 22, en la cual se realizaron los análisis estadísticos, empleando para ello la media, la desviación estándar (DS), la prueba de normalidad Kolmogorov-Smirnov (K-S), la t de Student, la U de Mann Whitney (transformados a una distribución Z), el ANOVA (F) y la prueba de Kruskall Wallis $\left(\mathrm{X}^{2}\right)$ para realizar la comparación por grupos, y el coeficiente no paramétrico Rho de Spearman para la correlación de variables.

\section{RESULTADOS}

En la tabla $\mathrm{N}^{\circ} 1$ se observa que el $50 \%$ de los docentes se ubican en un nivel alto de Agotamiento Emocional, 10,7\% en un nivel medio y el 39,3\% en un nivel bajo. El $78,6 \%$ de ellos presenta niveles bajos de Despersonalización, 5,4\% niveles medios y el $16,1 \%$ niveles altos. En cuanto a la Realización Personal, el 78,6\% se ubican en un nivel alto, $16,1 \%$ en un nivel medio y el $5,4 \%$ en un nivel bajo.

\section{Tabla No1}

Nivel de Agotamiento Emocional, Despersonalización y Realización Personal de la muestra total.

\begin{tabular}{lllllll}
\hline \multirow{2}{*}{ Niveles } & \multicolumn{2}{l}{$\begin{array}{l}\text { Agotamiento } \\
\text { Emocional }\end{array}$} & $\%$ & Despersonalización & \multicolumn{2}{c}{ Realización Personal } \\
\cline { 2 - 6 } & $\mathrm{n}$ & $\%$ & $\mathrm{n}$ & $\%$ & $\mathrm{n}$ & $\%$ \\
\hline Bajo & 22 & 39,3 & 44 & 78,6 & 3 & 5,4 \\
Medio & 6 & 10,7 & 3 & 5,4 & 9 & 16,1 \\
Alto & 28 & 50 & 9 & 16,1 & 44 & 78,6 \\
Total & 56 & 100 & 56 & 100 & 56 & 100 \\
\hline
\end{tabular}

En la tabla $\mathrm{N}^{\circ} 2$ se observa que el $46,4 \%$ de los docentes se sienten muy satisfechos, $30,4 \%$ satisfechos, $8,9 \%$ indiferentes, $10,7 \%$ insatisfechos y el 3,6\% muy insatisfechos con la vida.

\section{Tabla $\mathrm{N}^{\circ} 2$}

Nivel de Satisfacción con la vida de la muestra total.

\begin{tabular}{ccc}
\hline \multirow{2}{*}{ Niveles } & \multicolumn{2}{c}{ Satisfacción con la vida } \\
\cline { 2 - 3 } & $\mathrm{n}$ & $\%$ \\
\hline Muy Insatisfecho & 2 & 3,6 \\
Insatisfecho & 6 & 10,7 \\
Neutro o Indiferente & 5 & 8,9 \\
Satisfecho & 17 & 30,4 \\
Muy Satisfecho & 26 & 46,4 \\
Total & 56 & 100 \\
\hline
\end{tabular}


Se encontraron diferencias significativas según el sexo solo en la variable Despersonalización, en la cual los varones presentaron mayor puntaje que las mujeres (ver Tabla $\mathrm{N}^{\circ} 3$ ).

\section{Tabla $\mathbf{N}^{\circ} 3$}

Características y diferencias significativas de los componentes del Sindrome de Burnout y de la Satisfacción con la vida según el sexo.

\begin{tabular}{lllllllll}
\hline Variable & Grupos & Media & D.E. & K-S & $\mathrm{p}$ & Prueba estadística & $\mathrm{p}$ \\
\hline \multirow{2}{*}{ AE } & Varones & 21,35 & 13,200 &, 144 &, 200 & $\mathrm{t}$ & & \multirow{2}{*}{, 195} \\
& Mujeres & 26,14 & 13,045 &, 112 &, 200 & $-1,311$ & & \\
$\mathrm{D}$ & Varones & 6,05 & 6,597 &, $203^{*}$ &, 030 & $\mathrm{U}$ & $\mathrm{Z}$ & \multirow{2}{*}{, 032} \\
& Mujeres & 2,89 & 5,159 &, $288^{*}$ &, 000 & 238,5 & $-2,147^{*}$ & \\
\multirow{2}{*}{$\mathrm{RP}$} & Varones & 42,10 & 4,051 &, 140 &, 200 & $\mathrm{U}$ & $\mathrm{Z}$ & \multirow{2}{*}{, 336} \\
& Mujeres & 42,81 & 4,798 &, $156^{*}$ &, 028 & 304,0 &,- 962 & \\
\multirow{2}{*}{ SCLV } & Varones & 19,55 & 4,628 &, $239^{*}$ &, 004 & $\mathrm{U}$ & $\mathrm{Z}$ & \multirow{2}{*}{, 327} \\
& Mujeres & 18,78 & 4,058 &, 125 &, 171 & 303,0 &,- 979 & \\
\hline
\end{tabular}

${ }^{*} \mathrm{p}<.05 ; \mathrm{AE}=$ Agotamiento Emocional; $\mathrm{D}=$ Despersonalización; $\mathrm{RP}=$ Realización Personal; SCLV = Satisfacción con la Vida

No se encontraron diferencias significativas según la edad (ver Tabla $\left.\mathrm{N}^{\circ} 4\right)$.

\section{Tabla $\mathbf{N}^{\circ} 4$}

Características y diferencias significativas de los componentes del Sindrome de Burnouty de la Satisfacción con la vida según la edad.

\begin{tabular}{|c|c|c|c|c|c|c|c|}
\hline Variable & Grupos & Media & D.E. & K-S & $\mathrm{p}$ & $\begin{array}{l}\text { Prueba } \\
\text { estadística }\end{array}$ & $\mathrm{p}$ \\
\hline \multirow{4}{*}{$\mathrm{AE}$} & 24 a 30 años & 19,20 & 10,727 & 170 & 200 & & \multirow{4}{*}{, 491} \\
\hline & 31 a 40 años & 26,94 & 12,260 & 187 & 118 & $\mathrm{~F}$ & \\
\hline & 41 a 50 años & 23,78 & 14,240 &, 135 & 200 & ,815 & \\
\hline & Más de 50 años & 26,36 & 14,982 &, 140 & 200 & & \\
\hline \multirow{4}{*}{$\mathrm{D}$} & 24 a 30 años & 2,40 & 4,695 &, $334^{*}$ & ,002 & & \multirow{4}{*}{, 702} \\
\hline & 31 a 40 años & 5,06 & 7,232 &, $264^{*}$ & ,003 & $\mathrm{X}^{2}$ & \\
\hline & 41 a 50 años & 4,39 & 6,418 &, $252 *$ & ,004 & 1,415 & \\
\hline & Más de 50 años & 3,27 & 3,133 & ,216 & , 162 & & \\
\hline \multirow{4}{*}{$\mathrm{RP}$} & 24 a 30 años & 43,50 & 4,994 &, 184 & ,200 & & \multirow{4}{*}{, 073} \\
\hline & 31 a 40 años & 41,82 & 5,114 & ,201 &, 067 & $\mathrm{~F}$ & \\
\hline & 41 a 50 años & 41,06 & 4,263 & 144 & ,200 & 2,461 & \\
\hline & Más de 50 años & 45,27 & 1,794 &, 167 & ,200 & & \\
\hline \multirow{4}{*}{ SCLV } & 24 a 30 años & 18,80 & 4,492 & ,225 & ,163 & & \multirow{4}{*}{, 327} \\
\hline & 31 a 40 años & 17,65 & 4,182 &, 144 & ,200 & $\mathrm{X}^{2}$ & \\
\hline & 41 a 50 años & 19,78 & 4,250 & ,280* & 001 & 3,453 & \\
\hline & Más de 50 años & 20,27 & 4,027 &, 155 & 200 & & \\
\hline
\end{tabular}

${ }^{*} \mathrm{p}<.05 ; \mathrm{AE}=$ Agotamiento Emocional; D = Despersonalización; RP = Realización Personal; SCLV = Satisfacción con la Vida 
No se encontraron diferencias significativas según el tipo de Institución Educativa (ver Tabla $\mathrm{N}^{\circ}$ ).

\section{Tabla $\mathrm{N}^{\circ} 5$}

Características y diferencias significativas de los componentes del Sindrome de Burnouty de la Satisfacción con la vida según el tipo de Institución Educativa.

\begin{tabular}{|c|c|c|c|c|c|c|c|c|}
\hline Variable & Grupos & Media & D.E. & K-S & $\mathrm{p}$ & \multicolumn{2}{|c|}{ Prueba estadística } & $\mathrm{p}$ \\
\hline \multirow{2}{*}{$\mathrm{AE}$} & Nacional & 25,22 & 12,856 & ,132 & ,106 & \multirow{2}{*}{\multicolumn{2}{|c|}{$\begin{array}{c}t \\
620\end{array}$}} & 520 \\
\hline & Particular & 22,89 & 14,031 &, 163 & ,200 & & & ,538, \\
\hline \multirow{2}{*}{ D } & Nacional & 4,65 & 6,075 & ,234* & ,000 & U & Z & 100 \\
\hline & Particular & 2,79 & 5,350 &, $315^{*}$ & ,000 & 259,5 & $-1,645$ & 100 \\
\hline \multirow{2}{*}{$\mathrm{RP}$} & Nacional & 42,14 & 4,685 & ,168* & ,010 & $\mathrm{U}$ & Z & \multirow{2}{*}{,348 } \\
\hline & Particular & 43,37 & 4,179 &, $214^{*}$ & 022 & 297,5 &,- 939 & \\
\hline \multirow{2}{*}{ SCLV } & Nacional & 19,49 & 4,260 & ,179* & ,004 & $\mathrm{U}$ & Z & \multirow{2}{*}{, 326} \\
\hline & Particular & 18,21 & 4,198 &, 189 & 073 & 295,0 &,- 982 & \\
\hline
\end{tabular}

${ }^{*} \mathrm{p}<.05 ; \mathrm{AE}=$ Agotamiento Emocional; $\mathrm{D}=$ Despersonalización; $\mathrm{RP}=$ Realización Personal; SCLV $=$ Satisfacción con la Vida

No se encontraron diferencias significativas según el nivel educativo de enseñanza (ver Tabla $\mathrm{N}^{\circ} 6$ ).

\section{Tabla $\mathrm{N}^{\circ} 6$}

Características y diferencias significativas de los componentes del Sindrome de Burnout y de la Satisfacción con la vida según el nivel educativo de enseñanza.

\begin{tabular}{|c|c|c|c|c|c|c|c|}
\hline Variable & Grupos & Media & D.E. & K-S & $\mathrm{p}$ & Prueba estadística & $\mathrm{p}$ \\
\hline \multirow{3}{*}{$\mathrm{AE}$} & Inicial & 23,00 & 14,519 & 249 & 200 & \multirow{3}{*}{$\begin{array}{l}X^{2} \\
, 797\end{array}$} & \multirow{3}{*}{,671 } \\
\hline & Primaria & 27,00 & 13,204 & ,214* & 022 & & \\
\hline & Secundaria & 23,13 & 13,140 & ,119 & 200 & & \\
\hline \multirow{3}{*}{$\mathrm{D}$} & Inicial & 3,17 & 5,672 & ,378* &, 007 & \multirow{3}{*}{$\begin{array}{l}X^{2} \\
1,259\end{array}$} & \multirow{3}{*}{, 533} \\
\hline & Primaria & 4,16 & 6,842 & ,304* &, 000 & & \\
\hline & Secundaria & 4,10 & 5,412 & ,225* &, 000 & & \\
\hline \multirow{3}{*}{$\mathrm{RP}$} & Inicial & 42,50 & 5,505 & ,297 & ,106 & \multirow{3}{*}{$\begin{array}{l}X^{2} \\
, 128\end{array}$} & \multirow{3}{*}{,938 } \\
\hline & Primaria & 43,16 & 3,686 & ,117 & 200 & & \\
\hline & Secundaria & 42,19 & 4,888 & ,193* &, 005 & & \\
\hline \multirow{3}{*}{ SCLV } & Inicial & 18,33 & 6,022 & ,281 & ,151 & \multirow{3}{*}{$\begin{array}{l}X^{2} \\
, 197\end{array}$} & \multirow{3}{*}{,906 } \\
\hline & Primaria & 19,58 & 3,656 & ,220* & ,016 & & \\
\hline & Secundaria & 18,87 & 4,319 & ,152 & ,068 & & \\
\hline
\end{tabular}

${ }^{*} \mathrm{p}<.05 ; \mathrm{AE}=$ Agotamiento Emocional; $\mathrm{D}=$ Despersonalización; $\mathrm{RP}=$ Realización Personal; SCLV = Satisfacción con la Vida

Al realizar la correlación de variables (ver Tabla $\mathrm{N}^{\circ}$ ) se halló una relación significativa, media y positiva, entre la Despersonalización y el Agotamiento Emocional $(\mathrm{p}<.01, \mathrm{r}=, 510)$, una relación significativa, baja y negativa, entre la Realización Personal y la Despersonalización $(\mathrm{p}<.05, \mathrm{r}=-, 309)$, y una relación significativa, baja y positiva, entre la Satisfacción con la vida y la Realización Personal $(\mathrm{p}<.05, \mathrm{r}=$,323). 


\section{Tabla $\mathbf{N}^{\circ} 7$}

Análisis de Correlación (rho de Spearman) entre las dimensiones del Sindrome de Burnout y la Satisfacción con la Vida

\begin{tabular}{ccccc}
\hline Variables & $\begin{array}{c}\text { Agotamiento } \\
\text { Emocional }\end{array}$ & Despersonalización & $\begin{array}{c}\text { Realización } \\
\text { Personal }\end{array}$ & $\begin{array}{c}\text { Satisfacción con la } \\
\text { vida }\end{array}$ \\
\hline $\begin{array}{c}\text { Agotamiento } \\
\text { Emocional }\end{array}$ & 1 & & & \\
$\begin{array}{c}\text { Despersonali } \\
\text { zación }\end{array}$ &, $510^{* *}$ & 1 & & \\
$\begin{array}{c}\text { Realización } \\
\text { Personal }\end{array}$ &,- 247 &,$- 309 *$ & 1 & \\
$\begin{array}{c}\text { Satisfacción } \\
\text { con la vida }\end{array}$ &,- 120 &, 085 & $323^{*}$ & 1 \\
\hline $\mathrm{*}<<.05 ; * * \mathrm{p}<.01 ; \mathrm{N}=56$ & & &
\end{tabular}

\section{DISCUSIÓN}

El presente estudio tuvo como objetivo general encontrar una relación entre el Síndrome de Burnout y la Satisfacción con la vida. Para ello, se aplicaron los instrumentos respectivos a 56 docentes que realizaban clases virtuales en diversos departamentos del Perú debido a la pandemia por covid-19, hallándose finalmente una relación estadísticamente significativa, baja y positiva, entre la Realización Personal y la Satisfacción con la vida $(\mathrm{p}<.05, \mathrm{r}=, 323)$, lo cual indica que a mayor Realización Personal mayor Satisfacción con la vida tendrá el docente, y viceversa. Esto concuerda con la teoría planteada por Maslach (2009) y por Pavot y Diener (1993), quienes coinciden en el sentido de logro personal y profesional del individuo. De la misma forma con el modelo de la teoría Sociocognitiva del yo, la cual postula que el esfuerzo que dedique a sus actividades y el nivel de confianza que tenga el docente sobre sus propias capacidades, le permitirán tener mayores probabilidades de éxito en sus deberes (Gil-Monte y Peiró, 1999), aumentando su satisfacción laboral (Malander, 2016; Mengui, 2018) y su desempeño docente (Achiri, 2019; Nayda, 2018). Sin embargo, los hallazgos discrepan con lo obtenido por Cárdenas (2019) en Ayacucho, quien no encontró relaciones significativas entre los tres componentes del Síndrome de Burnout y la Satisfacción con la vida. Este contraste podría deberse a que en el presente estudio más de la mitad de la muestra son docentes que laboraban en Lima y solo uno laboraba en Ayacucho. Por lo cual una primera recomendación sería la de realizar un análisis comparativo según el lugar de trabajo (departamento donde labora), teniendo cantidades de sujetos similares en los diferentes grupos.

Al realizar el análisis descriptivo de los datos se encontró que el 50\% de los profesores que realizaban clases virtuales presentaban niveles altos de Agotamiento Emocional y un 10,7\% se ubicaba en un nivel medio. Según Maslach (2009) esta dimensión es el indicador por excelencia de la presencia del síndrome de Burnout, caracterizado por la sobreexigencia del mismo trabajador aún sin contar con las herramientas necesarias para afrontar dicha situación. Esto es alarmante, puesto que más de la mitad de docentes estaría en riesgo de desarrollar el síndrome. Lo cual afectaría negativamente en su desempeño laboral (Achiri, 2019), llevándole a sentirse insatisfecho 
con su trabajo (Arias et. al., 2017; Cordero y Vargas, 2019; Mengui, 2018). Lo hallado en el presente estudio difiere con lo reportado por Cárdenas (2019) y Pereira y Amacifen (2018) en Ayacucho e Iquitos, respectivamente, puesto que la mayoría de sus profesores evaluados presentaban niveles bajos de Agotamiento Emocional. Esta discordancia podría ser explicada por la localización de la muestra del presente estudio, pues la mayoría de profesores pertenecían a la región Costa. Por lo cual se afirma la primera recomendación.

Por otro lado, se halló que la mayor cantidad de docentes $(78,6 \%)$ presentaban niveles bajos de Despersonalización y niveles altos de Realización Personal. Lo cual quiere decir que a pesar de los bajos recursos emocionales de los docentes para afrontar la sobreexigencia en su trabajo, estos muestran resistencia a reaccionar negativamente ante sus alumnos (Maslach, 2009) y se perciben como personas competentes y autoeficaces (Mengui, 2018), favoreciendo de este modo su Desempeño Docente (Achiri, 2019; Nayda, 2018) y mostrándose empáticos con sus estudiantes y colegas de trabajo (Romero, 2019). Estos resultados son respaldados por los hallazgos de Cárdenas (2019) en Ayacucho, cuyos docentes evaluados presentaban altos niveles de Realización Personal y bajos niveles de Despersonalización. No obstante, estos hallazgos discrepan con lo encontrado por Pereira y Amacifen (2018) en Iquitos, quienes presentaban niveles altos de Despersonalización y niveles bajos de Realización Personal.

Con respecto a la Satisfacción con la vida, se halló que más del 75\% de docentes que realizaban clases virtuales durante la pandemia, se sentían entre satisfechos $(30,4 \%)$ y muy satisfechos con la vida (46,4\%). Estos resultados son respaldados por investigaciones hechas por Sylva (2019) en Ecuador, Cárdenas (2019) en Ayacucho, y Condori (2019) en Tacna. Por lo cual de deduce que los docentes del presente estudio tienden a lograr los objetivos que se plantean en la vida y en el trabajo, según sus propios criterios (Pavot y Diener, 1993).

A nivel comparativo, se realizó el análisis inferencial de los datos. Se encontró diferencias significativas, según el sexo, únicamente en la variable Despersonalización, en la cual los varones se muestran más indiferentes e insensibles con sus alumnos (Maslach, 2009) a comparación de las mujeres. Este dato es relevante puesto que se evidencia la importancia de las relaciones interpersonales de los docentes en la enseñanza-aprendizaje hacia sus estudiantes, en la cual las mujeres tienden a interactuar mejor con sus alumnos de forma virtual. Sin embargo, esto motiva a indagar también en las preferencias de los estudiantes según el sexo del docente al momento de las clases, de modo que ampliar su estudio en futuras investigaciones permitiría identificar con exactitud aquello que interfiere o beneficia en la interacción alumno-maestro. En las demás variables de estudio no se hallaron diferencias estadísticamente significativas, quiere decir que los profesores, tanto varones como mujeres, presentan niveles similares en el Agotamiento Emocional, la Realización Personal y la Satisfacción con la vida. Estos hallazgos contrastan con lo obtenido por Arias et. al. (2019) en Arequipa, quienes encontraron que los varones se sentían emocionalmente más agotados que las mujeres.

Con respecto a la edad, descriptivamente se observa que aquellos docentes con edades comprendidas entre 31 a 40 años puntúan más alto en las variables Agotamiento 
Emocional y Despersonalización, y que aquellos profesores que tienen más de 50 años de edad, puntúan alto en las variables Realización Personal y Satisfacción con la vida, sin embargo, estos resultados no son estadísticamente significativos $(p>.05)$. Lo hallado en el presente estudio discrepa con lo encontrado por Sylva (2019) en Ecuador, en la cual, a un nivel significativo, aquellas profesoras de educación Inicial que tenían mayor edad se sentían más satisfechas con la vida.

Según el tipo de Institución Educativa, descriptivamente se observa que aquellos docentes de colegios nacionales presentan mayor puntuación en las variables Agotamiento Emocional y Despersonalización que aquellos profesores de educación particular, más estos resultados no son estadísticamente significativos $(p>$.05). Lo cual discrepa con lo hallado por Arias y Jiménez (2013) en Arequipa, en donde, a un nivel significativo, los docentes de colegios nacionales presentaban mayor Despersonalización que los docentes de colegios particulares, además de que éstos últimos presentaban menor Realización Personal que aquellos que laboraban en colegios nacionales.

Con respecto al nivel educativo de enseñanza, descriptivamente se observa que los docentes de educación primaria presentan mayor puntuación en cada una de las variables de estudio, no obstante, estos hallazgos no son significativos ( $p>.05$ ). Estos resultados contrastan con lo obtenido en Arequipa por Arias et. al. (2019), quienes hallaron diferencias estadísticamente significativas en la variable Realización Personal, en la cual los docentes de educación Primaria se sentían más competentes y con mayor sentido de logro que los docentes de educación inicial y educación secundaria.

Cabe resaltar que la muestra del estudio fue de tamaño pequeño, debido a la poca disponibilidad de tiempo de los docentes y al modo de aplicación de los instrumentos, ya que algunos desconocían el uso del formulario. A pesar de ello, los análisis estadísticos permitieron obtener diferencias significativas según el sexo en la variable Despersonalización, y una relación significativa entre la Realización Personal y la Satisfacción con la Vida $(\mathrm{p}<.05)$, aunque de magnitud baja $(\mathrm{r}=, 323)$, por ello el resultado se ha de tomar con precaución. Por todo lo anterior, se propone realizar futuras investigaciones que amplíen los análisis del presente estudio, tomando en cuenta las recomendaciones planteadas líneas arriba.

\section{CONCLUSIONES}

- La mayor cantidad de docentes que realizan clases virtuales durante la pandemia por covid-19 presentan niveles altos en las variables Agotamiento Emocional (50\%) y Realización Personal (78,6\%), y niveles bajos en Despersonalización (78,6\%).

- La mayor cantidad de docentes se sienten satisfechos (30,4\%) y muy satisfechos con la vida (46,4\%).

- Según el sexo, se obtuvieron diferencias significativas $(\mathrm{p}<.05)$ solo en la variable Despersonalización.

- No se hallaron diferencias significativas $(\mathrm{p}>$.05) según la edad, el tipo de institución educativa y el nivel educativo de enseñanza.

- Existe una relación significativa, baja y positiva $(\mathrm{p}<.05 ; \mathrm{r}=$,323) entre la Realización Personal y la Satisfacción con la Vida. 


\section{REFERENCIAS BIBLIOGRÁFICAS}

Achiri, M. (2019). Síndrome de Burnout y el desempeño de los docentes de nivel secundario de la institución educativa Nro. 40046 José L. Cornejo Acosta, Acequia Alta Cayma Arequipa, 2018. Tesis de maestría. Universidad Nacional de San Agustín de Arequipa.

Aloe, A., Shisler, S., Norris, B., Nickerson, A., Tyler, R. (2014). A multivariate metaanalysis of student misbehavior and teacher Burnout. Educational Research Review. 12. Pp. 30-44. https://doi.org/10.1016/j.edurev.2014.05.003

Arias, W., Huamani, J., y Ceballos, K. (2019). Síndrome de Burnout en profesores de escuela y universidad: un análisis psicométrico y comparativo en la ciudad de Arequipa. Propósitos y Representaciones, 7(3), 72-110. http://dx.doi.org/10.20511 /pyr2019.v7n3.390

Arias, W., y Jiménez, N. (2013). Síndrome de burnout en docentes de Educación Básica Regular de Arequipa. Educación, 22(42), 53-76. http:// revistas.pucp.edu.pe/index. $\mathrm{php} /$ educacion/article/view/5291

Arias, W., Sánchez, M. y Ceballos, K. (2017). Síndrome de Burnout, satisfacción laboral y relaciones interpersonales en profesores de una institución educativa de Arequipa. Acta Psicológica peruana, 2(1), pp. 118-139. http://revistas.autonoma.edu.pe/ index.php/ACPP/article/view/72

Britez, M. (2020). La educación ante el avance del COVID-19 en Paraguay. Comparativo con países de la Triple frontera. Scielo Preprints. https://doi.org/10. 1590/SciELOPreprints.22

Calderón-De la Cruz, G., Lozano, F., Cantuarias, A., \& Ibarra, L. (2018). Validación de la Escala Satisfacción con la Vida en trabajadores peruanos. Liberabit, 24(2), 249-264. https://doi.org/10.24265/liberabit.2018.v24n2.06

Cárdenas, F. (2019). Relación de la satisfacción con la vida y el estrés laboral en docentes de la Institución Educativa Privada Saco Oliveros Ayacucho-2019. Tesis de Licenciatura. Universidad Católica Los Ángeles de Chimbote. http:// repositorio.uladech.edu.pe/handle/123456789/15130

Centro europeo para la prevención y el control de enfermedades (2020). Actualización de la situación de Covid-19 en todo el mundo. Recuperado de https://www.ecdc.europa.eu/en/geographical-distribution-2019-ncov-cases

Colomeischi, A. (2015). Teachers Burnout in Relation with Their Emotional Intelligence and Personality Traits. Procedia - Social and Behavioral Sciences. 180, pp 10671073. https://doi.org/10.1016/j.sbspro.2015.02.207

Condori, H. (2019). Satisfacción con la vida en docentes de instituciones educativas de un distrito de Tacna, 2019. Tesis de Licenciatura. ULADECH.

Cordero, P. y Vargas, A. (2019). Síndrome de Burnout y satisfacción laboral en docentes de Instituciones educativas estatales y privadas del distrito de San Martin de Porres. 
Tesis de licenciatura. Universidad Ricardo Palma. http://repositorio.urp.edu.pe/ handle/URP/2796

De Mendoza, A. (2020). Refundemos la escuela. El Peruano. Recuperado de https://elperuano.pe/noticia-refundemos-escuela-104463.aspx

Diener, Ed., Emmons, R., Larsen, R., \& Griffin, S. (1985). The Satisfaction With Life Scale. Journal of Personality Assessment, 49(1), 71-75. https://doi.org/10.1207/s15327752jpa4901_13

Fernández, M. (2002). Desgaste psíquico (burnout) en profesores de educación primaria de Lima metropolitana. Persona, (005), 27-66. https://doi.org/10.26439/ persona2002.n005.842

Gil-Monte, P.R. y Peiró, J.M. (1999). Perspectivas teóricas y modelos interpretativos para el estudio del síndrome de quemarse por el trabajo. Anales De Psicología / Annals of Psychology, 15 (2), 261-268. https://revistas.um.es/analesps/article/view/ 30161

González, N., Tejeda, A., Espinosa, C. y Oliveros, Z. (2020). Impacto psicológico en estudiantes universitarios mexicanos por confinamiento durante la pandemia por Covid-19. Preprints Scielo. https://doi.org/10.1590/SciELOPreprints.756

Hernández, R. (2017). Impacto de las TIC en la educación: Retos y Perspectivas. Propósitos y Representaciones, 5(1), 325 - 347. http://dx.doi.org/10.20511/ pyr2017.v5n1.149

Hidalgo, C. (06 de Mayo de 2020). Los maestros y la educación durante la pandemia. Archivo Covid-19 Perú. Consultado el 14 de Octubre de 2020. Recuperado de https://sisbib.unmsm.edu.pe/archivocovid19peru/test_audiovisual/testimonio_i nfo.php?id_ta $=1$

Instituto Nacional de Estadística e Informática (2017). Más de medio millón de maestros en el Perú celebran su día. Recuperado de https://www.inei.gob.pe/prensa/ noticias/mas-de-medio-millon-de-maestros-en-el-peru-celebran-su-dia-9833/\#: : text $=$ Con $\% 20$ motivo $\% 20 \mathrm{del}^{2} \% 20 \mathrm{D} \% \mathrm{C} 3 \% \mathrm{ADa} \% 20 \mathrm{del}, \mathrm{en} \% 20 \mathrm{el} \% 20$ sistema $\% 20 \mathrm{e}$ ducativo $\% 20$ nacional.

Lluch, T. (1999). Construcción de una escala para evaluar la Salud Mental Positiva. Tesis Doctoral. Universidad de Barcelona. España.

Malander, N. (2016). Síndrome de Burnout y Satisfacción Laboral en Docentes de Nivel Secundario. Ciencia y Trabajo. 18(57), 177-182.

Mariátegui, V. (06 de Mayo de 2020). Los maestros y la educación durante la pandemia. Archivo Covid-19 Perú. Consultado el 14 de Octubre de 2020. Recuperado de https://sisbib.unmsm.edu.pe/archivocovid19peru/test_audiovisual/testimonio_i nfo.php?id_ta $=7$

Maslach, C. (2009). Comprendiendo el Burnout. Ciencia y Trabajo, 11 (32), 37-43. 
Mateus, J. y Suárez-Guerrero, C. (2017). La competencia TIC en el nuevo currículo peruano desde la perspectiva de la educación mediática. Edmetic 6(2), 129-147. Recuperado de http://hdl.handle.net/10396/15404

Mengui, M. (2018). Teachers' Mental Health: Protective Factors. REVISTA ConCiencia EPG, 3(1), 13-30. Recuperado de https://ri.conicet.gov.ar/handle/11336/94528

Ministerio de Educación (2020). Aprendo en Casa. Recuperado de https://www. aprendoencasa.pe/\#/

Montes, W. (06 de Mayo de 2020). El rol de los maestros durante la pandemia. Archivo Covid-19 Perú. Consultado el 14 de Octubre de 2020. Recuperado de https://sisbib.unmsm.edu.pe/archivocovid19peru/test_audiovisual/testimonio_i nfo.php?id_ta $=3$

Morán, C., Vicente, F., Sánchez, A., y Montes, E. (2014) La psicología positiva: antigua o nueva concepción. Revista INFAD de Psicología. International Journal of Developmental and Educational Psychology., 3(1), 439-450. https://doi.org/10.17060/ijodaep.2014.n1.v3.522

Nayda, E. (2018). Síndrome de Burnout y su relación con el desempeño docente en el aula de una institución educativa particular, Arequipa - 2017. Tesis de licenciatura. Universidad Nacional de San Agustín.

Organización mundial de la salud (2020). Preguntas y respuestas sobre la enfermedad por coronavirus (COVID-19). Recuperado de https://www.who.int/es/emergencies/ diseases/novel-coronavirus-2019/advice-for-public/q-a-coronaviruses

Pavot, W., \& Diener, E. (1993). Review of the Satisfaction With Life Scale. Psychological Assessment, 5(2), 164-172. https://doi.org/10.1037/1040-3590.5.2.164

Pereira, I. y Amacifen, A. (2018). Nivel de Síndrome de Burnout en docentes de una institución educativa de la ciudad de Iquitos, Perú-2018. Tesis de Licenciatura. Universidad Científica del Perú - UCP.

Picón, G., Gonzáles, K. y Paredes, N. (2020). Performance y formación educativa en competencias digitales en clases no presenciales durante la pandemia covid-19. Scielo preprints. 1-22. https://doi.org/10.1590/SciELOPreprints.778

Quispe, M. (2020). La educación virtual: la gran oportunidad que ha dado el covid-19. Noticias Universidad Nacional Mayor de San Marcos. Recuperado de http://www.unmsm.edu.pe/noticias/ver/Educacion-virtual-la-gran-oportunidadque-ha-dado-el-COVID-19

Romero, K. (2019). Empatía y Síndrome de Desgaste Profesional (Burnout) en docentes de Educación Básica Regular de Lima Metropolitana. Avances en Psicología. 27 (2), 237-254. https://doi.org/10.33539/avpsicol.2019.v27n2.1801

Salgado, C. (2018). Manual de investigación. Teoría y práctica para hacer la tesis según la metodología cuantitativa. Lima: Fondo Editorial de la Universidad Marcelino Champagnat. 
Sandín, B., Valiente, R., García-Escalera, J., \& Chorot, P. (2020). Psychological impact of the COVID-19 pandemic: Negative and positive effects in Spanish people during the mandatory national quarantine. Journal of Psychopathology and Clinical Psychology, 25(1), 1-22. doi: https://doi.org/10.5944/rppc.27569

Skaalvik, E. M., \& Skaalvik, S. (2007). Dimensions of teacher self-efficacy and relations with strain factors, perceived collective teacher efficacy, and teacher burnout. Journal of Educational Psychology, 99(3), 611-625. https://doi.org/10.1037/ 0022-0663.99.3.611

Seligman, M. (2014). Flourish. A Visionary New Understanding of Happiness and Wellbeing. Ed. OCEANO. México.

Sylvia, M. (2019). La inteligencia emocional para la prevención y desarrollo emocional en la formación del profesorado de nivel de 3 a 5 años de educación inicial en Ecuador. Tesis doctoral. Universidad de Barcelona. http://diposit.ub.edu/dspace/bitstream /2445/144412/1/MYSL_TESIS.pdf

Vázquez, C., Duque, A., \& Hervás, G. (2013). Satisfaction with life scale in a representative sample of Spanish adults: validation and normative data. The Spanish Journal of Psychology. 16. https://doi.org/10.1017/sjp.2013.82

\section{CITAR COMO:}

Celio Pillaca, J. (2021). Burnout y satisfacción con la vida en docentes que realizan clases virtuales en un contexto de pandemia por covid-19. Puriq, 3(1), 104-119. https://doi.org/10.37073/puriq.3.1.142 